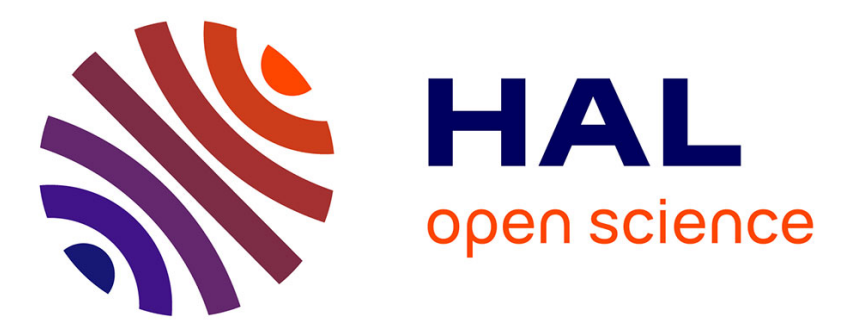

\title{
Diploid Cellular Automata: First Experiments on the Random Mixtures of Two Elementary Rules
}

\author{
Nazim A. Fatès
}

\section{To cite this version:}

Nazim A. Fatès. Diploid Cellular Automata: First Experiments on the Random Mixtures of Two Elementary Rules. AUTOMATA 2017 - 23th International Workshop on Cellular Automata and Discrete Complex Systems, Jun 2017, Milan, Italy. pp.97-108, 10.1007/978-3-319-58631-1_8 . hal01656351

\section{HAL Id: hal-01656351 \\ https://hal.inria.fr/hal-01656351}

Submitted on 5 Dec 2017

HAL is a multi-disciplinary open access archive for the deposit and dissemination of scientific research documents, whether they are published or not. The documents may come from teaching and research institutions in France or abroad, or from public or private research centers.
L'archive ouverte pluridisciplinaire HAL, est destinée au dépôt et à la diffusion de documents scientifiques de niveau recherche, publiés ou non, émanant des établissements d'enseignement et de recherche français ou étrangers, des laboratoires publics ou privés. 


\title{
Diploid cellular automata: first experiments on the random mixtures of two elementary rules
}

\author{
Nazim Fatès \\ Inria Nancy - Grand Est ; LORIA UMR 7503 \\ nazim.fates@loria.fr
}

\begin{abstract}
We study a small part of the 8088 diploid cellular automata. These rules are obtained with a random mixture of two deterministic Elementary Cellular Automata. We use numerical simulations to study the mixtures obtained with three blind rules: the null rule, the identity rule and the inversion rule. As the mathematical analysis of such systems is a difficult task, we use numerical simulations to get insights into the dynamics of this class of stochastic cellular automata. We are particularly interested in studying phase transitions and various types of symmetry breaking.
\end{abstract}

Keywords: stochastic cellular automata; probabilistic cellular automata; symmetry breaking; synchronisation

\section{Introduction}

As introduced by Turing in his article on morphogenesis [15], the question of randomness is fundamental to understand the laws of life. Turing was puzzled by the capacity of biological organisms to realise a form of "symmetry breaking": given a dynamical system which is invariant under some symmetry and an initial condition which is also invariant under this symmetry, such systems evolve to a state which is stable and which is no longer invariant under this symmetry. The typical case is the embryo which initially has a spherical form and needs to "break" this form to develop different organs. To produce such a phenomenon, two mechanisms can be at play: (a) the initial condition possesses a small asymmetry which is amplified in the evolution of the system; (b) the components of the system evolve with some randomness, which allows them to "choose" one of the symmetric directions of evolutions.

The framework of stochastic cellular automata is adapted for the study of such phenomena. In this note, we deal with one-dimensional cellular automata with random transitions, binary states and nearest neighbours. More specifically, we restrict our scope to the rules defined as a random mixture of two deterministic Elementary cellular automata (see the definitions below). As we will see, this case is sufficiently rich to provide many worthy examples, with potential applications in the study of physical, chemical or biological systems. We are particularly interested in the qualitative transformations that a cellular system may undergo when one progressively varies the mixing rate of its two deterministic components. 


\section{Definitions}

Elementary Cellular Automata. We consider finite cellular automata with binary states and periodic boundary conditions. We denote by $Q=\{0,1\}$ the set of states and let $\mathcal{L}=\mathbb{Z} / n \mathbb{Z}$ denote the set of $n$ cells arranged in a ring. A configuration represents the collection of states of all the cells at a given time step ; the set of configurations is denoted by $Q^{\mathcal{L}}$.

In this note, we are only interested in the systems where the cells update their state according to their own state and the state of their left and right neighbour. This set of three cells forms the neighbourhood of a given cell. At each time step, the updates are made synchronously according to a local rule $f: Q^{3} \rightarrow Q$. Given a local function $f$ and a set of cells $\mathcal{L}$, one can define the Elementary Cellular Automaton $F: Q^{\mathcal{L}} \rightarrow Q^{\mathcal{L}}$ such that, the image $y=F(x)$ of a configuration $x \in Q^{\mathcal{L}}$ is given by:

$$
\forall i \in \mathcal{L}, y_{i}=f\left(x_{i-1}, x_{i}, x_{i+1}\right) .
$$

An initial condition $x \in Q^{\mathcal{L}}$ is then associated to its trajectory, that is, the infinite sequence $\left(x^{t}\right)_{t \in \mathbb{N}}$ such that $x^{0}=x$ and $x^{t+1}=F(x)$ for all $t \in \mathbb{N}$.

Classically, a rule $f$ is identified by the decimal number $W$ which results from the conversion of the binary number $[f(1,1,1) f(1,1,0) \ldots f(0,0,0)]_{\mathrm{b}}$. (In other words $W(f)=f(0,0,0) \cdot 2^{0}+f(0,0,1) \cdot 2^{1}+\cdots+f(1,1,1) \cdot 2^{7}$.) It is common to identify the rule with its decimal code. When the ring size $n$ and the local rule $f$ are clear from the context, it is also common to identify the local rule with the Elementary Cellular Automaton (ECA) F.

We now turn our attention to stochastic cellular automata ${ }^{1}$. In this model, each cell has a probability to turn to 0 or to 1 according to the states of its neighbourhood. This means that we need to replace the trajectories $\left(x^{t}\right)_{t \in \mathbb{N}}$ by a random process $\left(\xi^{t}\right)_{t \in \mathbb{N}}$. Each $\xi^{t}$ is formed by the random variables $\left(\xi_{i}^{t}\right)_{i \in \mathcal{L}}$, which represent the random state of the cell $i$ at time $t$. Starting from given probability distribution $\xi^{0}$, the evolution of the stochastic cellular automaton is a Markov chain: intuitively this means that the knowledge of the state of the system at time $t$ is sufficient to calculate the probability distribution at time $t+1$. Since the state of each cell is calculated independently, we can use a function $\phi: Q^{3} \rightarrow[0,1]$, which gives the probability to be in state 1 if the neighbourhood of a cell is $(x, y, z)$. Starting from an initial random distribution $\xi^{0}$, the evolution of our stochastic cellular automaton can thus be obtained for each $t \in \mathbb{N}$ and for each $i \in \mathcal{L}$ with:

$$
\operatorname{Pr}\left\{\xi_{i}^{t+1}=1\right\}=\sum_{(x, y, z) \in Q^{3}} \operatorname{Pr}\left\{\left(\xi_{i-1}^{t}, \xi_{i}^{t}, \xi_{i+1}^{t}\right)=(x, y, z)\right\} \cdot \phi(x, y, z)
$$

An alternative way of defining the Markov chain $\left(x^{t}\right)_{t \in \mathbb{N}}$ is to specify, for two configurations $x, y \in Q^{\mathcal{L}}$, the probability to go from $x$ to $y$; we then have:

$\operatorname{Pr}\left\{\xi^{t+1}=y \mid \xi^{t}=x\right\}=\prod_{i \in \mathcal{L}} y_{i} \cdot \phi\left(x_{i-1}, x_{i}, x_{i+1}\right)+\left(1-y_{i}\right) \cdot\left(1-\phi\left(x_{i-1}, x_{i}, x_{i+1}\right)\right)$.

\footnotetext{
$\overline{1}$ The name probabilistic cellular automata is also frequently used and is a synonym.
} 
The formalism defined above is convenient for binary finite systems but it requires more elaborate definitions for infinite-size systems [8] or systems with more states or continuous states [2]. Note that other types of presentations of the rules may be used to facilitate the analysis [5,2].

Diploid ECA. Since a stochastic ECA $\phi$ is defined with eight probabilities, it is rather intuitive to try to understand how the combination of these eight probabilities influences the behaviour of a rule, for example if the rule may converge to a fixed point or not, and what is the average time of convergence. An important question is to determine what are the various "behaviours" that this space contains and how these behaviours are modified when one moves continuously in this space. Since this space already contains the 256 deterministic ECAs, which are difficult to understand theoretically (see e.g. [12]), one can easily guess that its generalisation to the space of probabilistic CA will generate a great number of new problems $[1,8]$.

In order to get some insights on this question, it is thus necessary to concentrate on a subset of the stochastic ECA space. We thus propose to focus on the stochastic mixtures of two deterministic ECA. Such rules have already been studied by many authors (e.g. [9]), but to our knowledge only in particular contexts. We want here to enlarge our view of this space of rules: as a first step, we examine some of their simple properties and explore their dynamics with numerical simulations.

We denote by $\mathcal{S}_{8}$ the set of stochastic ECA; this set is isomorphic to $[0,1]^{8}$ and for the sake of simplicity we will identify a stochastic rule and the function which maps each neighbourhood $(x, y, z) \in Q^{3}$ to its probability to update to state 1. Let $\phi \in \mathcal{S}_{8}$ be a stochastic ECA, we say that $\phi$ is a randomly-mixed $E C A$ if there exists two ECAs $f_{1}: Q^{3} \rightarrow Q$ and $f_{2}: Q^{3} \rightarrow Q$ and a constant $\lambda \in[0,1]$, called the mixing rate, such that:

$$
\forall(x, y, z) \in Q^{3}, \phi(x, y, z)=(1-\lambda) \cdot f_{1}(x, y, z)+\lambda \cdot f_{2}(x, y, z)
$$

We will write $\phi=\left(f_{1}, f_{2}\right)[\lambda]$ to denote this relation. When $f_{1} \neq f_{2}$ and $\lambda \in(0,1)$, we say that $\phi$ is a diploid $\mathrm{ECA}^{2}$.

It can be noted that not all randomly-mixed ECAs are diploids and that the decomposition of a diploid is not always unique. The following proposition clarifies this fact.

Proposition 1. Let $\phi$ be a randomly-mixed ECA, $\phi$ is a diploid if and only if there exists $\lambda \in(0,1)$ and $(x, y, z) \in Q^{3}$ such that $\phi(x, y, z)=\lambda$ and $\forall(x, y, z) \in$ $Q^{3}, \phi(x, y, z) \in\{0, \lambda, 1-\lambda, 1\}$.

Indeed, if $\phi$ is a diploid such that $\phi=\left(f_{1}, f_{2}\right)[\lambda]$ then there exists a triplet $(x, y, z) \in Q^{3}$ such that $\phi(x, y, z) \notin\{0,1\}$, which implies that $\lambda=\phi(x, y, z)$ or $\lambda=1-\phi(x, y, z)$. Moreover, it can be noted that if the mixing rate $\lambda$ is different

\footnotetext{
${ }^{2}$ The name is composed from the ancient Greek $\delta เ \pi \lambda o u ̃ s$ (diplous), which means twofold, double, and हi்os (eidos), which evokes the form, the shape, the face, etc.
} 
from $1 / 2$, then the decomposition of a diploid into its components $f_{1}$ and $f_{2}$ is unique, up to the exchange symmetry, that is, the symmetry that exchanges $f_{1}$ and $f_{2}$ and inverts $\lambda$ into $1-\lambda$.

Let $f_{1}$ and $f_{2}$ be two different ECAs, we call a diagonal the set of diploids that can be obtained by combining $f_{1}$ and $f_{2}$ with a mixing rate $\lambda \in(0,1)$. Diagonals can be seen as lying on the diagonals of the hypercube $[0,1]^{8}$, where the vertices of the hypercube represent the deterministic ECAs. They are simply denoted by $\left(f_{1}, f_{2}\right)=\left\{\left(f_{1}, f_{2}\right)[\lambda], \lambda \in(0,1)\right\}$.

Since there are $256 \mathrm{ECAs}$, there exist $256 \times 256=65536$ couples of ECAs that allow one to define a randomly-mixed ECA. If one is interested in diagonals then there exist $256 \cdot(256-1) / 2=32640$ couples to study, taking into account the exchange symmetry. However, the conjugation symmetry, which exchanges the $0 \mathrm{~s}$ and $1 \mathrm{~s}$, and the reflection symmetry, which exchanges the left and right directions, can also be employed to reduce the number of couples that one may study to explore the space of diploid rules. Let us now enumerate the number of couples that are non-equivalent according to these symmetries.

Let $R, C, T$ be the operators which respectively represent the reflection, the conjugation, and the composition of both operations. Formally for an ECA $f$ : $Q^{3} \rightarrow Q$, we define:

$$
\begin{aligned}
& R(f)=f(z, y, x), \\
& C(f)=1-f(1-x, 1-y, 1-z), \\
& T(f)=R \circ C(f)=C \circ R(f)=1-f(1-z, 1-y, 1-x) .
\end{aligned}
$$

It is straightforward to generalise the definitions above to the stochastic rules, either by applying the symmetries to the local function or by applying them to the configurations that define the Markov chain of the ECA (see above).

Proposition 2. Under the symmetries $R, C$, and $T$, there are 8808 non-equivalent diagonals that define the diploid rules.

Proof. Let B be the set of ECAs which are invariant under both symmetries $R$ and $C$ (and thus $T$ ), let R, C, T be respectively the ECAs which are invariant under only the symmetry $R, C$, or $T$ only and let $\mathrm{N}$ represent the rules which have no invariance. It can be easily verified that we have $|\mathrm{B}|=2^{3}=8,|\mathrm{C}|=$ $|\mathrm{T}|=2^{4}-|\mathrm{B}|=8,|\mathrm{R}|=2^{6}-|\mathrm{B}|=56$ and $|\mathrm{N}|=256-(|\mathrm{B}|+|\mathrm{R}|+|\mathrm{C}|+|\mathrm{T}|)=176$.

Let $b, r, c, t, n$ represent the number of classes of equivalences of the sets $\mathrm{B}$, $\mathrm{R}, \mathrm{C}, \mathrm{T}$ and N, respectively. We have $b=|\mathrm{B}|=8, r=|\mathrm{R}| / 2=28, c=|\mathrm{C}| / 2=4$, $t=|\mathrm{T}| / 2=4$ and $n=|\mathrm{N}| / 4=44$. The total number of non-equivalent ECAs is thus equal to $b+c+r+t+n=88$, we represent each class of equivalence by the rule which has the smallest decimal code, the minimal representative.

We say that a couple of rules $(f, g)$ is invariant under the symmetry $S$ if (a): $(f, g)=(S(f), S(g))$ or (b): $(f, g)=(S(g), S(f))$. Unfortunately, it is not possible to obtain the equivalence classes of the diagonals by only considering the couples formed by the minimal representatives and the exchange symmetry. For example, if one considers the two ECAs 0 and 255, 0 is a minimal representative but not 255, but, obviously, it is not possible to form a couple of two minimal representative rules that would generate an equivalent diagonal. 
Let $\mathrm{S}=Q^{Q^{3}}$ denote the ECA space and $\mathrm{S}_{\mathrm{d}}=\mathrm{S} \times \mathrm{S}$ be the space of couples of ECAs. Taking similar notations as we did for the ECA space, let $B_{d}$ be the set of couples that are invariant under both symmetries $R$ and $C$, and $\mathrm{R}_{\mathrm{d}}, \mathrm{C}_{\mathrm{d}}$ and $\mathrm{T}_{\mathrm{d}}$ the set of couples that are invariant under only the symmetry $R, C$ and $T$, respectively, and let $\mathrm{N}_{\mathrm{d}}$ be the set of couples that have no invariance. Note that $\mathrm{S}_{\mathrm{d}}$ contains 256 repetitive couples, that is, couples in the form $(f, f)$.

Invariance by $\mathbf{R}$ and $\mathbf{C}$. The set $\mathrm{B}_{\mathrm{d}}$ is formed by the couples $(f, g)$ such that: $(1)(f, g)=(R(f), R(g))$ or $(2)(f, g)=(R(g), R(f))$; and such that: (3) $(f, g)=(C(f), C(g))$ or $(4)(f, g)=(C(g), C(f))$. Let $\mathrm{B}_{\mathrm{d}}^{1}$ be the set of couples which verify (1) and (3), we have $\left|\mathrm{B}_{\mathrm{d}}^{1}\right|=|\mathrm{B} \times \mathrm{B}|=8 \cdot 8=64$. Let $\mathrm{B}_{\mathrm{d}}^{2}$ be the set of couples which verify (1) and (4) and which do not belong to $B_{d}^{1}$. It can be easily verified that $\mathrm{B}_{\mathrm{d}}^{2}=\{(f, g), f \in \mathrm{R}, g=C(f)\}$, which gives $\left|\mathrm{B}_{\mathrm{d}}^{2}\right|=|\mathrm{R}|=56$. Let $\mathrm{B}_{\mathrm{d}}^{3}$ be the set of couples which verify (2) and (3) and which do not belong to $\mathrm{B}_{\mathrm{d}}^{1}$. Similarly, it can be easily verified that $\mathrm{B}_{\mathrm{d}}^{3}=\{(f, g), f \in \mathrm{C}, g=R(f)\}$, which gives $\left|\mathrm{B}_{\mathrm{d}}^{3}\right|=|\mathrm{C}|=8$. In a similar way: $\mathrm{B}_{\mathrm{d}}^{4}=\{(f, g), f \in \mathrm{T}, g=R(f)=C(f)\}$, which gives $\left|\mathrm{B}_{\mathrm{d}}^{4}\right|=|\mathrm{T}|=8$.

One may easily verify that the sets $B_{d}^{1}, B_{d}^{2}, B_{d}^{3}, B_{d}^{4}$ form a partition of $B_{d}$. We thus have: $\left|\mathrm{B}_{\mathrm{d}}\right|=64+56+8+8=128+8=136$. Given that $\mathrm{B}_{\mathrm{d}}$ contains 8 repetitive couples and given that each pair of non-repetitive couples $(f, g)$ and $(g, f)$ counts for one diagonal, the number of diagonals that can be generated by elements of $B_{d}$ is $\#\left(B_{d}\right)=(136-8) / 2=64$.

Invariance by $R$ only. The set $R_{\mathrm{d}}$ is formed by the couples such that: (1) $(f, g)=(R(f), R(g))$ or $(2)(f, g)=(R(g), R(f))$, and such that: $(3)(f, g) \notin \mathrm{B}_{\mathrm{d}}$.

Let $\mathrm{R}_{\mathrm{d}}^{1}$ be the set of couples that verify condition (1) and not condition (2), and let $R_{d}^{2}$ be the set of couples that verify condition (2) but not condition (1). We have: $R_{d}^{1}=(B \cup R) \times(B \cup R)$, which gives $\left|R_{d}^{1}\right|=64 \cdot 64=4096$. We have: $\mathrm{R}_{\mathrm{d}}^{2}=\left\{(f, g) \in \mathrm{S}_{\mathrm{d}}, f \in \mathrm{S} \backslash(\mathrm{B} \cup \mathrm{R}), g=R(f)\right\}$, which gives: $\left|\mathrm{R}_{\mathrm{d}}^{2}\right|=256-(56+8)=$ 192. As $R_{d}=\left(R_{d}^{1} \cup R_{d}^{2}\right) \backslash B_{d}$, we find that $\left|R_{d}\right|=4096+192-136=4152$. Given that $\mathrm{R}_{\mathrm{d}}$ contains $|\mathrm{R}|=56$ repetitive couples, and given that each quadruplet $(f, g),(g, f),(C(f), C(g)),(C(g), C(f)$ counts for one non-equivalent diagonal, we have: $\#\left(\mathrm{R}_{\mathrm{d}}\right)=(4152-56) / 4=4096 / 4=1024$.

Invariance by $C$ only and by $R \circ C$ only. The same arguments as seen above can be repeated. We count: $\left|\mathrm{C}_{\mathrm{d}}\right|=\left|\mathrm{T}_{\mathrm{d}}\right|=256+240-136=360$. The number of non-equivalent diagonals found in $\mathrm{C}_{\mathrm{d}}$ and $\mathrm{T}_{\mathrm{d}}$ is: $\#\left(\mathrm{~T}_{\mathrm{d}}\right)=\#\left(\mathrm{C}_{\mathrm{d}}\right)=88$

No invariance. We now enumerate the number of couples which show no invariance by $\mathrm{R}, \mathrm{C}$ or $R \circ C$. We have $\mathrm{N}_{\mathrm{d}}=\mathrm{S}_{\mathrm{d}} \backslash\left(\mathrm{B}_{\mathrm{d}} \cup \mathrm{R}_{\mathrm{d}} \cup \mathrm{C}_{\mathrm{d}} \cup \mathrm{T}_{\mathrm{d}}\right)$ and we count: $\left|\mathrm{N}_{\mathrm{d}}\right|=256.256-(136+4152+360+360)=65536-5008=60528$. Since this set of couples contains $|\mathrm{N}|=256-(8+56+8+8)=176$ repetitive couples and since the exchange symmetry and the symmetries $\mathrm{C}, \mathrm{R}$ or $R \circ C$ allow one to associate 8 different couples in one equivalence class, we find that the number of non-equivalent diagonals that can be generated by elements of $\mathrm{N}_{\mathrm{d}}$ is $\#\left(\mathrm{~N}_{\mathrm{d}}\right)=(60528-176) / 8=7544$.

We are now in position to add the set cardinals calculated above to find how many non-equivalent diagonals there are to generate all the possible diploids: $\#\left(\mathrm{~S}_{\mathrm{d}}\right)=64+1024+88+88+7544=8808$. 
To introduce an order on these 8808 couples of diploid generators, we may simply use the lexicographic order on the pair of decimal codes attached to each rule. In other words, given two pairs of $\operatorname{rules}(f, g)$ and $\left(f^{\prime}, g^{\prime}\right)$ we say that $(f, g) \leq\left(f^{\prime}, g^{\prime}\right)$ if and only if $f<f^{\prime}$ or if $f=f^{\prime}$ and $g \leq g^{\prime}$. As seen above, the couples may be grouped by sets of equivalent pairs of size 2 (elements of $B_{d}$ ), 4 (elements of $\mathrm{R}_{\mathrm{d}}, \mathrm{C}_{\mathrm{d}}, \mathrm{T}_{\mathrm{d}}$ ), or 8 (elements of $\mathrm{N}_{\mathrm{d}}$ ). We call a minimal representative the pair which has the smallest code in its class of equivalence.

\section{First steps in the space of diploids}

As already mentioned, there exists a plethora of interesting questions that can be asked on stochastic cellular automata. As a first step, we propose to examine a simple question: given a diagonal $\left(f_{1}, f_{2}\right)$, what kind of qualitative change may happen to the diploids of this diagonal when $\lambda$ is varied? In particular, we want to know if there exists some values of $\lambda$ where the behaviour of the system changes drastically. Here again, the answer we give may vary widely depending on how we estimate the "behaviour" of our cellular automaton. We thus simply propose to use two macroscopic parameters: the density, the ratio of the states 1 and the kinks density, the ratio of the occurrence of the length-2 patterns 01 and 10.

The exhaustive study of the 8808 diagonals is a daunting task, we thus need to focus on some families of diploids. We propose to begin our examination with the diagonals that are formed with the most simple rules: the null rule, the identity rule, and the inversion rule.

\subsection{Experimental protocol}

For a given ring size $n$ and a configuration $x \in Q^{\mathcal{L}}$, we call the density the ratio $d(x)=|x|_{1} / n$ where $|x|_{1}=\left|\left\{i \in \mathcal{L}, x_{i}=1\right\}\right|$ and kinks density the ratio $d_{\mathrm{k}}(x)=\left(|x|_{10}+|x| x_{01}\right) / n$ where $|x|_{q q^{\prime}}=\mid\left\{i \in \mathcal{L}, x_{i}=q\right.$ and $\left.x_{i+1}=q^{\prime}\right\} \mid$. For each couple of rules $(f, g)$ we will sample the value of $d$ and $d_{\mathrm{k}}$ of the stochastic mixtures of two ECAs for a system composed of $n=10000$ cells. We vary $\lambda$ from 0 to 1 by increasing $\lambda$ from 0 to 0.05 by steps of 0.01 , then from 0.05 to 0.95 by steps of 0.05 , and then from 0.95 to 1 by steps of 0.01 . The step length is made smaller for high and small values of $\lambda$ to give a more precise view close to the special points $\lambda=0$ and $\lambda=1$, where important modifications may occur. Indeed, the transition from a deterministic system to a non-deterministic one is likely to produce an abrupt change of behaviour; however, we leave for future work the study of such effects and we concentrate on the changes which occur for non-extremal values, as they are in some sense more surprising. For each diploid, we start from a uniform initial condition where each cell has an equal probability to be in state 0 or 1 ; we let the system evolve during $T_{\text {trans }}=5000$ time steps and we sample the value of the parameters $d$ and $d_{\mathrm{k}}$ to have a numerical estimation of the asymptotic density, that is, the average value we would obtain if the system's size would tend to infinity and if the sampling operation was repeated an infinite number of times. We sample only one value of this density for each value of $\alpha$. 

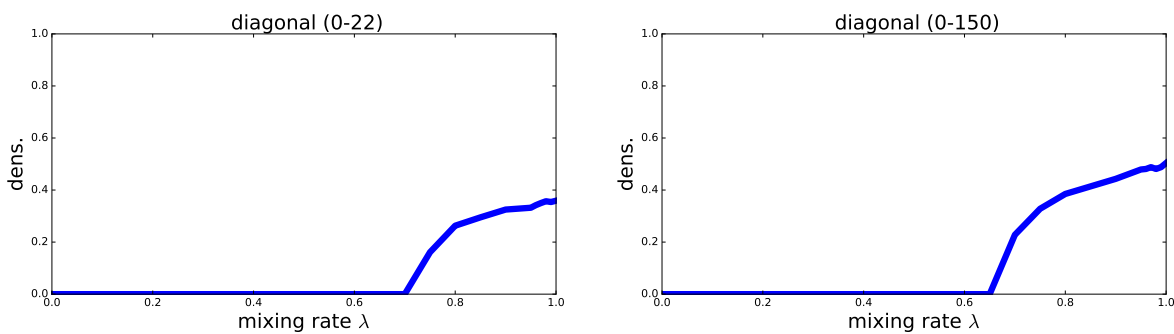

Fig. 1: Density as a function of $\lambda$ for the diagonals $(0,22)$ and $(0,150)$.

3.2 Mixtures with the null rule (ECA 0)

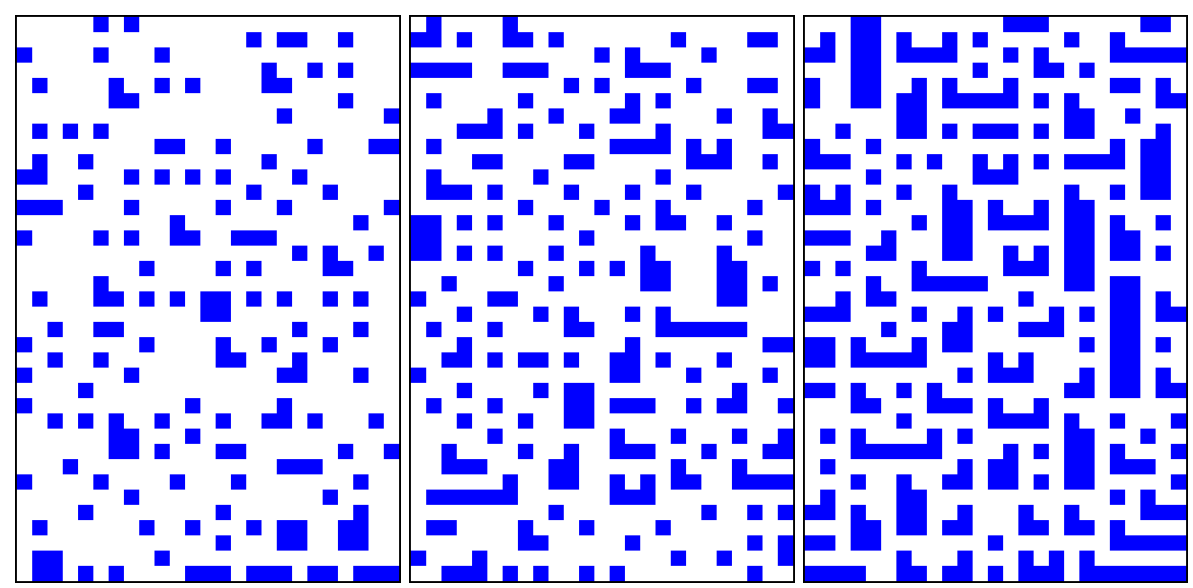

Fig. 2: Space-time diagrams of diploids $(0,73)$ with (left): $\lambda=0.25$, (middle): $\lambda=0.50$, right: $\lambda=0.75$. Time goes from bottom to top. Cells in state 0 and 1 are respectively represented by squares in white or blue (or dark gray).

We applied the protocol described above for the 159 minimal non-equivalent diagonals in the form $(0, f)$. Figure 1 shows the estimation of the asymptotic density as function of the mixture rate in two diagonals where a qualitative modification of the behaviour occurs. This type of brutal change of behaviour is well-known and is called a second-order phase transition: the function $d(\lambda)$ is continuous but its derivative is not. There exists a critical mixing rate $\lambda_{c}$ which separates a behaviour where the system converges to $0^{\mathcal{L}}$ (passive phase) and a behaviour with a stationary non-zero density (active phase). If the system were composed of an infinite number of cells, the property stated above would be exact. However, because of finite-size effects, it should be noted that even for the active phase, there exists a small but non-zero probability to reach the absorbing 
state $0^{\mathcal{L}}$, for example if all the cells apply rule 0 at the same time step. Regnault studied these type of phenomenon of convergence and he gave analytical results for a particular $\alpha$-asynchronous ECA rule (see below) [10]. Second-order phase transitions were detected in no less than 34 diagonals $(0, f)$ and where $f$ is found in the following ECA list: $18,22,26,28,30,50,54,58,60,62,78,90,94,110$, $122,126,146,150,154,156,158,178,182,186,188,190,202,206,218,22,234$, 238, 250, 254 (see also Tab. 1 p. 11).

It can be noted that the diagonals $(0,250)$ and $(0,254)$ correspond to two cases of oriented site percolation: in this problem an infinite grid is formed of sites which can be open with probability $p$ and closed with probability $1-p$ and starting from a particular site, a fluid flows from open site to open site in a given direction. There exists a critical threshold for $p$ for which the fluid percolates, that is, has the possibility to travel arbitrary distances in the medium. The critical threshold has been measured with Monte Carlo simulations and various analytical estimates have been proposed. One may report to the study by Taggi [14] for more details and for a more general view on such percolation operators with various neighbourhoods.

We did not observe any phase transitions within the diagonals formed by ECA 0 and the ECAs with an odd code, that is, the rules for which $f(0,0,0)=1$. Figure 2 shows the space-time diagrams of three diploids taken in the diagonal $(0,73)$ with $\lambda \in\{0.25,0.5,0.75\}$. These samples allow one to understand why the mixtures between rule 0 and an "odd" rule does not create any interesting structure: on the one hand, the null rule has a tendency to remove the $1 \mathrm{~s}$ independently on any information on the neighbourhood. On the other hand, the odd rule may create a 1 in any place where there are three successive 0s. This idea needs of course to be studied with more attention and if possible by analytical means.

\subsection{Mixtures with the identity rule : $\alpha$-asynchronous ECA}

The diploids defined with a mixture of the identity rule (ECA 204) and another ECA rule correspond to the $\alpha$-asynchronous CA (where the mixing rate $\lambda$ corresponds to the synchrony rate $\alpha$ ). Such a family of rules has been studied quite in detail both numerically and analytically but there are still many challenges in their understanding $[3,6,13,4]$. The most surprising aspect of $\alpha$-asynchronous $\mathrm{CA}$ is their great diversity of behaviour. There are 9 diagonals which contain a second-order phase transition for the density, but, contrarily to "mixed-with-0" diploids, it is not possible to predict the respective positions of the absorbing and active phases. For example, for ECA 50, the active phase is for high values of $\alpha$ and the absorbing phase is for low values, while the situation is inverted for ECA 6 or ECA 138. We refer to our survey on asynchronous for more information on this topic and on closely-related questions [6]. 

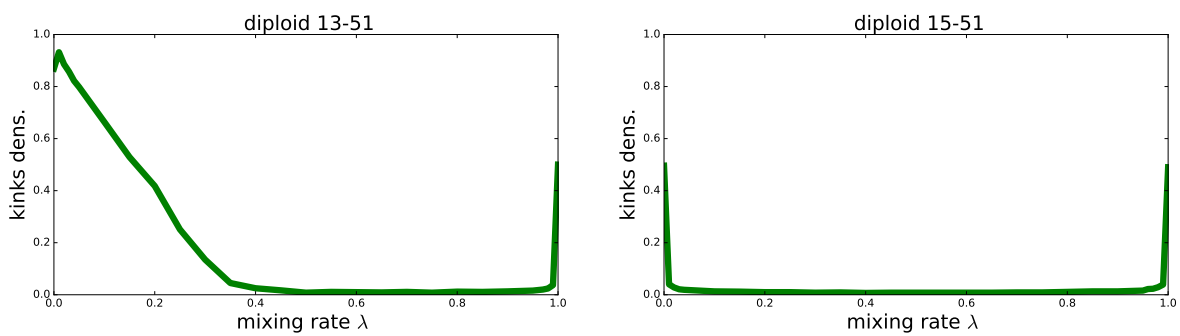

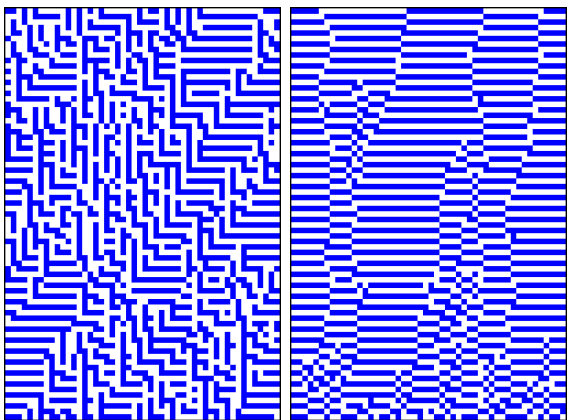

$(13,51)[0.20]$

$(13,51)[0.80]$

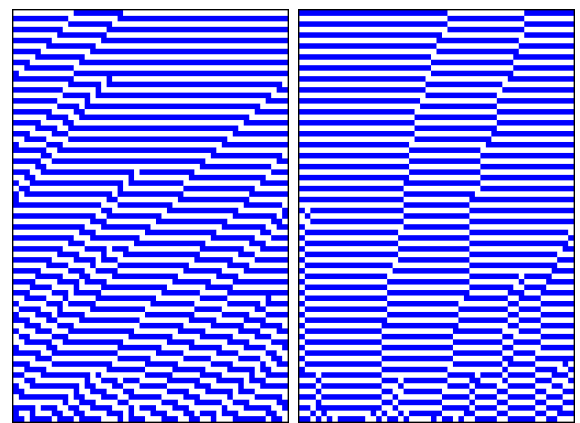

$(15,51)[0.20]$

$(15,51)[0.80]$

Fig. 3: Analysis of the diagonals $(13,51)$ and $(15,51)$. (top) Kinks density as a function of $\lambda$; (bottom) space-time diagrams for two arbitrary values of $\lambda$.
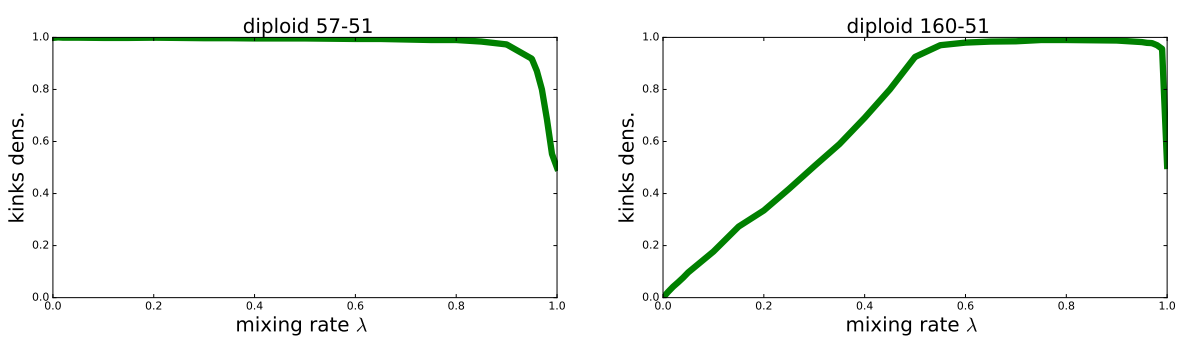

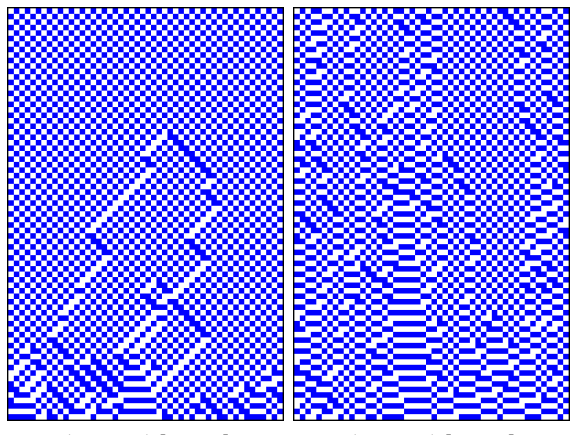

$(57,51)[0.25]$

$(57,51)[0.75]$

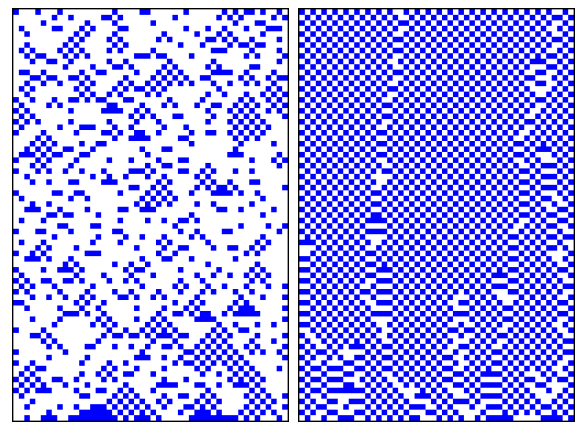

$(160,51)[0.25]$

$(160,51)[0.75]$

Fig. 4: Analysis of the diagonals $(57,51)$ and (160,51). (top) Kinks density as a function of $\lambda$; (bottom) space-time diagrams for two arbitrary values of $\lambda$. 


\subsection{Mixtures with the inversion rule (ECA 51)}

An interesting family of diploids to study is formed by the rules obtained as a mixture with ECA 51, that is, the rule $f(x, y, z)=1-y$. Contrarily to the previous families of diagonals, we did not observe any phase transition related to the evolution of the density. However, a new behaviour appears: there are some rules for which the kinks density take extremal values and become close (or equal) to 0 or 1.

First, let us examine the cases where the kinks density is close to 0. This happens in 16 diagonals $(f, 51)$ for the rules $f$ which have the following codes: 1 , $3,5,7, \mathbf{9}, 11, \mathbf{1 3}, 15,25,27,29,33,37, \mathbf{4 5}, \mathbf{7 3}$, and 77 . The diagonals formed with rules written in bold font show the presence of a critical threshold: they converge to a zero kink value only for sufficiently high values of $\lambda$ (see also Tab. 1). The other rules seem to remove the kinks for any value of the mixing rate. A peculiar behaviour appears for the diagonal $(33,51)$ where the kinks disappear very slowly for high values of $\lambda$. Experiments do not show a critical phenomenon here. Fig. 3 displays the evolution of the kinks density for the diagonals $(13,51)$ and $(15,51)$.

We represented examples of the space-time diagrams of the four diploids: $(13,51)[.2],(13,51)[.8],(15,51)[.2]$, and $(15,51)[.8]$ in Fig. 3,. The space-time diagrams of the diploids which remove the kinks show that the qualitative behaviour of these rules is quite regular: they tend to form blinking regions of consecutive 0s and 1s. This blinking phenomenon is joined to a random walk of the frontiers. The regions disappear when their frontiers meet (annihilation phenomenon) which allows to infer that all these diploids solve the global synchronisation problem [7]: from any initial condition, they converge to the deterministic period-2 cycle where the uniform configurations $0^{\mathcal{L}}$ and $1^{\mathcal{L}}$ alternate. Interestingly, Richard has shown that it is not possible to solve this problem with a deterministic cellular automaton (see Ref. [11] and the extended version of Ref. [7]), but as it can be seen here, solving the problem with diploids is rather easy. It can be shown analytically that the convergence time of the diploid $(15,51)$ scales quadratically with the number of cells. The proof of this property relies on a coupling with the shift rule (ECA 170) with an $\alpha$-asynchronous updating [7].

Let us now turn our attention on the diagonals which show values of the kinks density which are close or equal to 1 . There are 15 such diagonals $(f, 51)$ where $f$ has the code: 32, 34, 40, 42, 56, 57, 58, 104, 106, 122, 160, 162, 168, 170, 232. The rules written in bold font show the presence of a critical threshold: they converge to configurations where the states 0 and 1 alternate (high kinks density) only for a sufficiently high values of the mixing rate $\lambda$. The other diagonals seem to contain diploids which attain a kinks density of 1 for any value of $\lambda \in(0,1)$. The diagonal $(57,51)$ shows a slowdown of the convergence for high values of $\lambda$.

As it can be seen on Fig. 4, this behaviour can be associated to another form of symmetry breaking: the creation of a pattern where all the cells alternate their state in space and time. Here again, the synchronisation of the whole system is achieved by the random movements of the frontiers between non-synchronised regions, which act as defects which annihilate when they meet. 
Table 1: List of diagonals where second-order phase transitions were observed. The first column shows the order parameter for which the critical phenomenon is observed, the second and third columns show the two elements of the diagonal.

\begin{tabular}{|c|c|c|}
\hline param. & diagonal with & rule list \\
\hline dens. & 0 (null) & $\begin{array}{l}18,22,26,28,30,50,54,58,60,62,78,90,94,110, \\
122,126,146,150,154,156,158,178,182,186,188, \\
190,202,206,218,22,234,238,250,254\end{array}$ \\
\hline dens. & 204 (identity) & $6,18,26,38,50,58,106,134,146$ \\
\hline kinks dens. & 51 (inversion) & $9,13,32,40,45,73,104,106,122,160,168,232$ \\
\hline
\end{tabular}

\section{Discussion}

This note constitutes a first step in the exploration of the space of the diploids. Such mixtures of two deterministic rules have been studied by various authors and it would be interesting to compile and "digest" the various phenomena that were studied on such models. Here, we systematically explored the mixtures formed with three "blind" rules which do not depend on their neighbourhood: the null rule, the identity rule and the inversion rule, and obtained some quite puzzling observations.

First, in the space of diploids, the occurrence of second-order phase transitions is not a rare phenomenon. So far, similar observations were linked to percolation phenomena [14], to the study of the effects of asynchronism [6], or rely on specific constructions. Note that if one takes a "randomly" chosen rule in the space of the elementary stochastic rules $\mathcal{S}_{8}$, this rule is most likely a positive-rate rule, that is, for each neighbourhood there is a non-zero probability to reach both states. As a consequence, the chances to observe a phase transition by slightly modifying this rule is quite small (see Ref. [8] for more explanations). By contrast, the space of diploids may prove an appropriate subset of rules to find various forms of critical phenomena.

The second interesting aspect observed with our diploids is their ability to produce various forms of symmetry breaking: their inherent randomness allow them to easily create patterns with alternating states, or to reach homogeneous and synchronised blinking configurations. So far, diploids have provided simple solutions to the density classification problem (Fuks' rule, Schüle's rule and the traffic-majority rule) [5] or to the global synchronisation problem [7]. It is an open question to know how such models may perform some more complex forms of decentralised computations, and, if possible, solve problems that would be difficult or impossible to solve without the help of randomness ${ }^{3}$.

\footnotetext{
3 The author is grateful to Victor Roussanaly for his work on this topic as an intern in the Loria laboratory from June to August 2013. He also expresses his sincere recognition to Jordina Francès de Mas for her attention when reading the manuscript.
} 


\section{References}

1. Pablo Arrighi, Nicolas Schabanel, and Guillaume Theyssier. Stochastic cellular automata: Correlations, decidability and simulations. Fundamenta Informaticae, 126(2-3):121-156, 2013.

2. Witold Bołt, Jan M. Baetens, and Bernard De Baets. On the decomposition of stochastic cellular automata. Journal of Computational Science, 11:245 - 257, 2015. arXiv:1503.03318.

3. Alberto Dennunzio, Enrico Formenti, Luca Manzoni, and Giancarlo Mauri. masynchronous cellular automata: from fairness to quasi-fairness. Natural Computing, 12(4):561-572, 2013.

4. Alberto Dennunzio, Enrico Formenti, Luca Manzoni, Giancarlo Mauri, and Antonio E. Porreca. Computational complexity of finite asynchronous cellular automata. Theoretical Computer Science, 664:131 - 143, 2017.

5. Nazim Fatès. Stochastic cellular automata solutions to the density classification problem - when randomness helps computing. Theory of Computing Systems, 53(2):223-242, 2013.

6. Nazim Fatès. A guided tour of asynchronous cellular automata. Journal of Cellular Automata, 9(5-6):387-416, 2014.

7. Nazim Fatès. Remarks on the cellular automaton global synchronisation problem. In Jarkko Kari, editor, Proceedings of AUTOMATA 2015, volume 9099 of Lecture Notes in Computer Science, pages 113-126. Springer, 2015. extended version available on https://members.loria.fr/nazim.fates/dwnld/ submitted-GlobalSynchProblem-Fates17.pdf.

8. Jean Mairesse and Irène Marcovici. Around probabilistic cellular automata. Theoretical Computer Science, 559(0):42-72, 2014. Non-uniform Cellular Automata.

9. J. Ricardo G. Mendonça and Mário J. de Oliveira. An extinction-survival-type phase transition in the probabilistic cellular automaton p182-q200. Journal of Physics A: Mathematical and Theoretical, 44(15):155001, 2011.

10. Damien Regnault. Proof of a phase transition in probabilistic cellular automata. In Marie-Pierre Béal and Olivier Carton, editors, Proceedings of Developments in Language Theory, volume 7907 of Lecture Notes in Computer Science, pages 433444. Springer, 2013.

11. Gaétan Richard. On the synchronisation problem over cellular automata. To appear. Private communication, 2017.

12. Martin Schüle and Ruedi Stoop. A full computation-relevant topological dynamics classification of elementary cellular automata. Chaos, 22(4):043143, 2012.

13. Fernando Silva, Luís Correia, and AndersLyhne Christensen. Modelling synchronisation in multirobot systems with cellular automata: Analysis of update methods and topology perturbations. In Georgios Ch. Sirakoulis and Andrew Adamatzky, editors, Robots and Lattice Automata, volume 13 of Emergence, Complexity and Computation, pages 267-293. Springer International Publishing, 2015.

14. Lorenzo Taggi. Critical probabilities and convergence time of percolation probabilistic cellular automata. Journal of Statistical Physics, 159(4):853-892, 2015.

15. Alan Turing. The chemical basis of morphogenesis. Philosophical Transactions of the Royal Society (London), 237:5-72, 1952. 